Ágora Rev. Cient. 2019; 06(01):e3

\title{
Percepción de la enfermera en el arte de cuidar
}

\author{
Perception of nurse in the art of care
}

Rosa Reina ${ }^{1}$, María Hilda Cardenas ${ }^{1}$

\section{RESUMEN}

Introducción: El cuidado de enfermería como arte exige destrezas particulares, conocimiento y capacidades para establecer vínculos significativos, orientar el curso apropiado de la acción cuidadora, dando sentido a lo cotidiano, para satisfacción del paciente. Objetivo: Comprender la percepción de la enfermera al arte de cuidar; un aporte epistemológico que contribuya a fortalecer la práctica profesional y el componente del conocimiento estético que envuelve el objeto material de Enfermería, el arte de cuidar. Materiales y Métodos: La técnica de recolección de la información por entrevista en profundidad y la observación interpretativa, a enfermeras de los servicios de Medicina, Cirugía y Gineco-obstetricia, que aceptaron participar en el estudio, guardando la confidencialidad convenida en el consentimiento informado. El aporte teórico-explicativo fue determinado por dos (2) categorías: El arte de cuidar es conocimiento y la forma de hacer las cosas y El cuidado individual basado en las necesidades. Resultados: Como consideraciones de cierre se tiene que el estudio del arte de cuidar permitió comprender el significado que le asignan al arte de cuidar en su desempeño cotidiano un grupo de profesionales de enfermería y de pacientes, además de comprender el sentido global de la práctica del cuidado y su complejidad. Todo lo cual nos permite asumir y sentirnos protagonistas como agentes de cambio de los modos de cuidar de nuestra profesión. Conclusión: La investigación del cuidado de enfermería se enriquece con la producción del conocimiento, que la enfermera puede asumir y emplear para fortalecer el papel de cuidadora como acompañante en los procesos de desarrollo y crecimiento de la civilidad humana.

Palabras claves: percepción, enfermera, arte, cuidar

\begin{abstract}
Introduction: Nursing care as art requires particular skills, knowledge and skills to establish meaningful links, guide the appropriate course of caregiving action, giving meaning to the everyday, for patient satisfaction. Objective: To understand the nurse's perception of the art of caring; an epistemological contribution that contributes to strengthen the professional practice and the component of the aesthetic knowledge that surrounds the material object of Nursing, the art of caring. Materials and Methods: The technique of gathering information by in-depth interview and interpretive observation, to nurses of the Medicine, Surgery and obstetric and gynecological services, which agreed to participate in the study, keeping the confidentiality agreed upon in the informed consent. The theoretical-explanatory contribution was determined by two (2) categories: The art of caring is knowledge and the way of doing things and Individual care based on needs. Results: As closure considerations, the study of the art of caring allowed to understand the meaning assigned to the art of caring for a group of nursing and patient professionals in their daily performance, as well as to understand the overall meaning of the practice of care and its complexity All of which allows us to assume and feel protagonists as agents of change in the ways of taking care of our profession. Conclusion: Nursing care research is enriched with the production of knowledge, which the nurse can assume and use to strengthen the role of caregiver as a companion in the processes of development and growth of human civility.
\end{abstract}

Keywords: perception, nurse, art, caring

${ }^{1}$ Universidad de Carabobo. Facultad de Ciencias de la Salud. Escuela de Enfermería. Venezuela.

\section{INTRODUCCIÓN}

Históricamente, la enfermería ha existido como actividad desde el inicio de la humanidad, pues siempre han existido personas con problemas de salud y enfermedad, que han ameritado de los cuidados de otras personas con la disposición de cuidar. Boff, afirma: "Sin cuidado, los seres humanos no podrían existir. No se concibe la vida misma si no existiera el cuidado. Sin cuidado, la 
persona pierde su estructura, pierde sentido y muere. Este hecho marca la existencia humana y muestra el verdadero origen del cuidado, el cual al estar influido por la cultura, es diverso, plural y universal" (1).

Se dice que el cuidado de enfermería como arte exige destrezas particulares, conocimiento y capacidades para establecer vínculos significativos, orientar el curso apropiado de la acción cuidadora, dando sentido a lo cotidiano, para satisfacción del paciente. Ciertamente, el arte del cuidado es la aplicación de destrezas particulares de la ciencia de enfermería. Sin embargo, su verdadera esencia creadora y estética se manifiesta en la relación interpersonal enfermera/persona cuidada durante la aplicación de técnicas y procedimientos, donde se vinculen la reflexión, la integración de creencias y valores, el análisis, el conocimiento, el juicio crítico y la intuición para crear y recrear el cuidado.

La concepción de enfermería como el arte de cuidar viene desde su origen como profesión, a mediados del siglo XIX, cuando nuestra pionera Florence Nightingale sostuvo: "La enfermería es un arte, y si se pretende que sea un arte, requiere una devoción tan exclusiva, una preparación tan dura, como el trabajo de un pintor o de un escultor; pero, ¿cómo puede compararse la tela muerta o el frío mármol con tener que trabajar con el cuerpo vivo, el templo del espíritu de Dios? Es una de las bellas artes; casi diría, la más bella de las Artes" (2).

Las enfermeras de hoy, tienen la oportunidad y la autonomía profesional de cumplir objetivos mediante herramientas de cuidado esencial para dar seguridad, proteger la salud física, psicológica y espiritual, en aras de evitar complicaciones para el paciente.

Comportamientos de cuidado que generen empatía, confianza, y socialización, con acciones basadas en conocimientos, su pericia en la comunicación, orientación, información, educación al paciente, son indispensables para manejar con arte, más allá de la técnica y del conocimiento un contexto de cuidado integral que respeta la dignidad humana de la persona. Wrubel y Benner afirman que: "el cuidado es esencial para el ejercicio eficaz de la enfermería. La enfermería nunca puede reducirse a la mera técnica y al conocimiento científico, porque el humor, el enojo, el cariño, la administración de medicamentos e incluso la enseñanza del paciente tienen distintas consecuencias en un contexto cuidador frente a otro que no lo es" (3).

Las autoras en su concepción contemplan otros componentes del cuidado como una característica inherente a la práctica de enfermería en la cual las enfermeras ayudan a los clientes de manera integral a recuperarse frente a la enfermedad, a darle sentido a esa situación para mantener la conexión con un contexto cuidador. El cuidar permite dar cuidado, hace que las enfermeras detecten cuales son las intervenciones que tienen éxito y este compromiso es el que guía la selección de cuidados presentes y futuros.

Sin embargo, en el contexto actual de la salud nos encontramos en oportunidades y contextos, por no decir en todos los servicios públicos, con condiciones de trabajo deplorables que no incentivan el trabajo creativo e interactivo, no hay espacio ni recursos para la creatividad sino para la improvisación de tareas, que no por ser muchas se hacen pesadas, sin gracia y sin desenvolvimiento. En ambientes en esas condiciones el trabajo se hace algunas veces, tan solo como actividad de producción, contrario a lo pregonado por Aristóteles "que todo el mundo produce, pero no todos tienen arte". Además, basado puramente en necesidades y no en la interacción como la acción política del cuidado, lo cual en el pensamiento de Arendt, es la actividad de la condición humana que conecta a la gente con el mundo humano (4).

Un ambiente agradable, sin tantas carencias, hace posible saber qué hacer con y cómo estar en el momento, surge de la percepción directa de lo que es importante del encuentro. La percepción del significado de un encuentro crea acciones artísticas de enfermería, y la percepción del significado del encuentro se refleja en las acciones independientes que emprende (5).

Como objetivo de estudio se planteó: Comprender la percepción de la enfermera al arte de cuidar. El cuidado enfermero es un arte que une lo tecnológico, intelectual y creativo con lo espiritual, cada individuo es único e irrepetible, la enfermera al darle cuidado lo crea de acuerdo con las necesidades de cada paciente, sin subordinarse a lo mecánico, para lograrlo, requiere una interrelación de personas con personas y no entre piezas de una maquinaria en la que la enfermera transfiera su propio ser a la función que hace.

\section{MATERIALES Y MÉTODOS}

La investigación es un diseño de tipo cualitativo por ser un proceso de estudio con abordaje del fenómeno en su entorno natural de trabajo, donde el investigador no solo está interesado en observar para conocer el fenómeno, sino que va más allá, en virtud de que mantiene una conversación abierta, cara a cara con los actores sociales para comprender el significado que estos le asignan al fenómeno en base a lo que 
dicen y hacen, siendo el investigador el principal instrumento de la recolección de la información con la cual interactúa para la generación de teoría a partir de los datos en bruto. El investigador va en busca de la experiencia del otro a través del diálogo para indagar aspectos que no pueden ser medidos en números absolutos, tales como, sentimientos, valores, creencias, ideas, emociones y todo aquello que guarda relación con la interacción humana. El interés es descubrir, interpretar y comprender cuales de estos aspectos que se dicen en las teorías del cuidado como arte, tienen significado en la práctica de interacción enfermera/ persona cuidada. Con la orientación filosófica del Interaccionismo Simbólico y el enfoque metodológico de la Teoría Fundamentada en los datos, con el propósito de Comprender la percepción de la enfermera al arte de cuidar. La técnica de recolección de la información por entrevista en profundidad y la observación interpretativa, a enfermeras de los servicios de Medicina, Cirugía y Gineco-obstetricia

\section{RESULTADOS Y DISCUSIÓN}

Como resultado de la presente investigación surgieron dos (2) categorías

\section{El arte de cuidar es conocimiento y la forma de hacer las cosas}

Para las enfermeras entrevistadas, cuando se les pregunto cómo percibes tú el arte de cuidar, esto fue lo que respondieron:

Yo considero que el arte de cuidar es el conocimiento y la forma de hacer las cosas, las técnicas que yo aplique las hice como debe ser, el arte es que uno sienta lo que quiere hacer para que la persona sienta que lo estoy haciendo con amor(liligar)...tú crees que lo importante es dominar la técnica, en cambio, el trato con el enfermo es lo realmente importante, no le dedicamos tiempo, a escuchar, a verlos, algunos pacientes se sienten aliviados con una palmadita((Taló).

Según la fenomenología, la percepción es una modalidad original de la conciencia, toda percepción se presenta dentro de cierto horizonte y en el mundo. Para las enfermeras del estudio, la percepción del arte de cuidar es variada y puede considerarse lógico, pues cada persona tiene su visión del mundo. Para alguien del grupo consiste en tener conocimiento, en la forma de hacer las cosas, que sienta lo que quiere hacer, visualiza que la persona esté satisfecha y perciba que lo está cuidando con amor, expresan que las técnicas son necesarias, pero el trato con el enfermo es lo realmente importante, pero no le dedican tiempo a escuchar, a verlo o hablar.

Se dice que el arte presupone tres elementos fundamentales. La técnica, la intuición y la sensibilidad. El arte no está en decir algo, está en hacerlo bien. Al interpretar los relatos de las informantes, encontramos que liligar expresa esas tres cualidades, aunque no expresa literalmente la palabra intuición, si expresa la palabra conocimiento y la intuición es un tipo de conocimiento que aparece de súbito en la mente humana. La intuición es la visión o la comprensión directa e inmediata de la realidad, sin elementos que se interpongan.

Pero es que además, el arte siempre ha sido uno de los principales medios de expresión del ser humano, a través del cual manifiesta sus ideas y sentimientos, la forma como se relaciona con el mundo. Hoy día se entiende por artista a alguien que practica las bellas artes. Aún así, el término artista puede tener diversas acepciones, desde el artista como creador, hasta el artista que tiene en la práctica, el arte de su profesión (6). En el caso de este análisis, compartimos con la definición actual de artista, pues desde sus orígenes como profesión, Nightingale calificó la enfermería como "la más bella de las artes" y la enfermera ha creado y ha hecho de su práctica del cuidado, el arte de su profesión, el arte de cuidar. Además, el arte como medio de expresión del ser humano, a través del cual manifiesta sus ideas y sentimientos, la forma como se relaciona con el mundo, también en (liligar) encontramos su percepción de ideas y sentimientos al expresar: el arte es que uno sienta lo que quiere hacer para que la persona sienta que lo estoy haciendo con amor, no solamente está expresando su sentir, sino también considerando los sentimientos del paciente: que sienta que lo estoy cuidando con amor. Para sustentar lo interpretado, Boff señala: todo comienza con el sentimiento. Es el sentimiento el que nos hace sensibles al otro, nos une a las cosas y nos envuelve con las personas. Ese sentimiento profundo es lo que se llama cuidado (1) y continua, el dato originario no es el logo, la razón o las estructuras de comprensión, sino el phatos, el sentido, la capacidad de simpatía y empatía, la dedicación, el cuidado y la comunicación con lo diferente.

Dice Souriau, al artista se le supone una disposición especialmente sensible frente al mundo que lo rodea, desarrollando su propio punto de vista, su creatividad, una buena técnica y un medio de comunicación hacia el espectador por medio de sus obras (7). Parafraseando a Souriau, 
diríamos que en ese referente teórico, la enfermera como artista en el arte de cuidar, posee las cualidades supuestas por el autor, una disposición especialmente sensible frente al paciente y el mundo que la rodea. El cuidado cotidiano le permite desarrollar su propia visión del mundo, su creatividad, el dominio de las técnicas de enfermería y la relación terapéutica como medio de comunicación con el paciente, quien es el espectador de su obra, la forma de cuidarlo.

Por su parte (Talo) percibe que en el arte de cuidar la técnica es importante, pero lo más importante es la relación con el enfermo, expresa en pocas palabras: el trato con el enfermo es lo realmente importante, no le dedicamos tiempo, a escuchar, a verlos, a hablar. Es obvio que Talo se refiere a la relación enfermera/paciente. Percibe que debido al estado de alteración física, o de dependencia, la persona enferma espera del equipo de enfermería, el cuidado que atienda todas sus necesidades. En esa perspectiva, considera la importancia del conocimiento que la enfermera debe tener de la técnica, pero enfatiza que lo realmente importante en el arte de cuidar es el trato con el paciente. Al decir de Boff, el cuidado significa desvelo, solicitud, diligencia, celo, atención, buen trato. Estamos ante una actitud fundamental donde la persona sale de sí y se centra en el otro con desvelo y solicitud. Por su propia naturaleza, el cuidado encierra dos significados básicos ligados entre sí. El primero, la solicitud de desvelo, de solicitud y de atención para con el otro. Y, segundo, de preocupación y de inquietud, porque la persona que tiene a su cuidado se siente envuelta y afectivamente ligada al otro (1).

Dentro de eso percibe que hay que dedicarle tiempo, a la escucha, a la mirada y al tacto, para compartir quizás los temores por el cambio de su mundo de los objetos, cambios de rutinas que envuelve la enfermedad. Entre ellos, la separación de la familia, el encontrarse en un ambiente y personas desconocidas, los ruidos del hospital, todas estas son señales de comunicación que la enfermera ha de tener habilidad de interpretar para comprender, porque a veces la hospitalización se vuelve un período estresante. Blumer afirma: en el mundo de los objetos es que los seres humanos desarrollan sus acciones. Para entender las acciones de las personas, es necesario identificar su mundo de los objetos. Los objetos deben ser vistos como creaciones sociales. La vida y la acción de las personas cambian con los cambios de su mundo de objetos (8). De manera que es lógica la percepción de Talo al decir que no se le dedica tiempo a la relación interpersonal enfermera/paciente. La relación persona a persona es una interacción de dos o más seres humanos, de modo que ambos puedan compartir sus sentimientos, valores, significados mediante el encuentro comunicacional. La comunicación constituye un fundamento cultural de la persona humana más que eso, de la propia vida. Es una necesidad básica, sin la cual la existencia de la humanidad sería imposible.

Según Travelbee, la relación enfermera/paciente es una relación persona a persona, con la intención de auxiliar a la persona en la comprensión del dolor y sufrimiento vivido. Vemos en la relación enfermera/paciente las personas en acción, es una relación de satisfacer las necesidades del paciente, donde la enfermera escoge los métodos eficaces de ayuda, envolviendo no solo a la persona enferma, sino todo lo que le rodea (9). Talo en su relato reconoce la falta de dedicación de tiempo al trato con el paciente: no le dedicamos tiempo, a escuchar, a verlos, a hablar. El tiempo y el espacio son categorías fundamentales en el acto de cuidar. Más aun, el arte de cuidar supone un tiempo, un tiempo de dedicación, un tiempo de continuidad, una característica del cuidado es que es continuo, no solamente es importante cuidar, sino también cuanto y donde cuidar. El arte de cuidar como cualquier arte requiere tiempo y dedicación. Cuidar de alguien no es un acto instantáneo, es un proceso que se desenvuelve en una cadena de instantes, de encuentros, de miradas (6). El profesional responsable padece la situación de la falta de tiempo para dialogar, pues el sistema no le permite cuidar dialógicamente, más aún su labor profesional se pondera por la rapidez, por su eficacia y cantidad y no por la eficiencia y la calidad. La enfermera se encuentra atrapada entre la masificación de personas enfermas en un mismo espacio, con escasez de personal y la consecuente falta de tiempo, luce contradictorio con lo recomendado por la norma, que a juicio de Balderas (10), los criterios para cuidados parciales (hospitalización sin gravedad, recuperación) son de 3 a 4 enfermeras por cada turno. Actualmente, es sabido de todos, las carencias tanto de recursos humanos como materiales de los hospitales, lo cual va en detrimento del cuidado de la salud.

\section{El cuidado individual basado en las necesidades}

Cuando se les pregunto a las enfermeras cómo percibes tú el arte de cuidar, esta fue su respuesta:

Cada paciente es un mundo, yo debo individualizar el cuidado, saber que necesidades tiene uno diferente del otro, brindar cuidados no solo físicos sino emocionales, espirituales $y$ 
psicológicos, brindarle cuidado que lo ayuden a recuperarse (Juren)...ayudar al paciente en la satisfacción de sus necesidades básicas, alimentación, hidratación, respiración e higiene, escuchar y hablar con el paciente, acompañarlo en el proceso de recuperar su salud, enseñarle para que pueda hacerlo por sí mismo (Talo)

Juren y Talo expresan su percepción del arte de cuidar con diferencias textuales, pero al analizarlas coinciden en su significado con características comunes, muy congruentes que a la vez pueden conceptualizarse como: cuidado individualizado, basado en las necesidades humanas.

El proceso de cuidar a una persona sana o enferma solo puede ser óptimo si es individual, porque cada persona humana es un ser único e irrepetible. Significa que las entrevistadas están claras en lo que respecta al cuidado individual, se intuye que basadas en esa individualidad, es que perciben el arte de cuidar basado en las necesidades del paciente, cuando expresan: saber que necesidades tiene uno diferente del otro. De hecho, perciben al paciente desde una concepción holística, puesto que consideran el cuidado en su dimensión física, emocional y espiritual. La unicidad del hombre es evidente y se manifiesta en el mundo a través del lenguaje, de la expresión, de su cuerpo y de su rostro. Por todo eso es imposible cuidar en masa o en grupo, porque cada ser humano tiene su propia realidad y cuidarlo en su enfermedad es apostar a su singularidad (4).

La teoría de las necesidades humanas: propone los conceptos de que el cuidado está centrado en satisfacer las necesidades humanas. Entre las autoras de estas teorías se encuentra: Virginia Henderson, Dorotea Orem, Faye Abdellah y Nancy Roper. Estos modelos aplican conceptos de la teoría de la psicología humanística, formulada por Abrahan Maslow, quien centra su propuesta en definir las necesidades humanas como unidades de motivación personal, organizadas en jerarquía dispuesta en una pirámide de cinco (5) niveles: Fisiológicas Básicas, Seguridad, Afiliación, Autoestima y Autorrealización.

La enfermera como persona experta en proporcional cuidado a la población, basa su actividad en cubrir las necesidades del individuo en todas las dimensiones del ser humano. El concepto del ser humano como un todo indivisible ha proporcionado unos conceptos a las enfermeras para dirigir su práctica al cuidado integral individualizado. El cuidado desde la percepción individual presupone gran conocimiento personal, de su circunstancia, biológica y biográfica. Mayeroff. Afirma: Para cuidar de otro, es preciso conocer principalmente quien es el otro, cuáles son sus poderes y limitaciones, cuáles son sus necesidades y lo que conduce a su crecimiento, preciso saber cómo responder a sus necesidades y cuáles son mis propios poderes y limitaciones (11). Ese conocimiento tiene que ser general y específico.

El discurso de las enfermeras entrevistadas, además de estar relacionado con la tesis de Mayeroffk, revela también la característica fundamental de los modelos que pertenecen a la escuela de las necesidades, que definen la acción de la enfermera como la persona que ayuda en la satisfacción de las necesidades fundamentales de la persona o de sus capacidades para el auto cuidado.

En la asistencia centrada en la satisfacción de las necesidades humanas, la enfermera reconoce las necesidades biofísicas, psicofísicas, psicosociales e interpersonales de ella misma y del paciente. Los pacientes deben satisfacer las necesidades de orden inferior antes de intentar satisfacer las de orden superior.

La alimentación, la eliminación y la ventilación son ejemplos de necesidades biofísicas de orden inferior, mientras que la actividad/ inactividad y la sexualidad se consideran necesidades psicofísicas de orden inferior. La realización de la persona y las relaciones interpersonales son necesidades psicosociales de orden superior. La autorrealización es una necesidad intra e interpersonal de orden superior. De allí que las informantes les dan prioridad y orden a las necesidades básicas.

Se interpreta en este discurso, que no solo se corresponde con la teoría de las necesidades de Maslow, sino que a su vez cave perfectamente dentro de la jerarquía de las necesidades humanas, ubicándolas en el nivel inferior de la pirámide. Maslow, quien es uno de los principales representantes de la escuela de psicología humanística, defiende en su teoría que los seres humanos están en continuo crecimiento y desarrollo desde su concepción hasta la muerte. Las necesidades motivan la conducta del individuo, la persona tiene que satisfacer sus necesidades fisiológicas y de seguridad (nivel inferior) para pasar a satisfacer otras más elevadas (nivel superior) como el amor, la autoestima, para llegar al final de la pirámide donde se sitúa la necesidad de autorrealización (12). Una de las teóricas de enfermería que trabaja el modelo de las escuela de las necesidades es Virginia Henderson, quien define a la enfermería en términos funcionales: "La única función de una enfermera es ayudar al individuo sano o enfermo, en la 
realización de aquellas actividades que contribuyan a su salud, su recuperación o una muerte tranquila, que éste realizaría sin ayuda si tuviese la fuerza, la voluntad y el conocimiento necesario. Y hacer esto de tal forma que le ayude a ser independiente lo antes posible" (4).

Partiendo de la teoría de las necesidades humanas básicas, la autora identifica catorce (14) necesidades básicas y fundamentales que comporten todos los seres humanos, que pueden no satisfacerse por causa de una enfermedad o en determinadas etapas del ciclo vital, incidiendo en ellas factores físicos, psicológicos o sociales.

Juren percibe el arte de cuidar con una visión integral, lo cual pone de manifiesto en su verbo de manera muy expresa: brindar cuidados no solo físicos sino emocionales, espirituales $y$ psicológicos. Aquí volvemos a encontrar su coincidencia con la visión holística del paciente. Cada vez somos más conscientes de que el conocimiento de la salud no se reduce a algo puramente biológico, sino que afecta a toda la persona. Por eso todas las intervenciones en salud han de tener también una perspectiva holística, global e integral.

Para Virginia Henderson, la persona es una unidad corporal/física y mental, que está constituida por componentes biológicos, psicológicos, sociológicos y espirituales. La mente y el cuerpo son inseparables. El paciente y su familia son considerados como una unidad (4). Cuidar en sentido holístico significa entonces considerar a las personas en todas sus dimensiones, es decir en la dimensión física, intelectual, social, emocional, espiritual y religiosa. En enfermería podemos decir que humanizar la salud es generar salud holística, en efecto, es uno de los indicadores del cuidado humanizado, es la consideración de la persona ayudada en sentido holístico, en toda su corporeidad.

La naturaleza intangible y variable de la espiritualidad, variedad de formas en que se experimenta entre diferentes personas y en distintos momentos, las múltiples formas que tiene a través del cuerpo, del pensamiento, de los sentimientos, los juicios y la creatividad, y su relación con los aspectos no materiales de la vida, explican en parte esta percepción.

La espiritualidad agrupa las ideas filosóficas del sentido de la vida y su propósito, y tiene el poder de dar forma y significado al ser, del saber $\mathrm{y}$ del hacer que se puede reconocer como un impulso unificador para el bienestar humano que motiva para escoger las relaciones y búsquedas necesarias. De allí que Talo percibe en su visión de salud holística, la espiritualidad como una necesidad del paciente hospitalizado que debe ayudarlo a satisfacer, estableciendo una relación de encuentro enfermera-persona cuidada con presencia autentica. Para admitir que un profesional cuida y valora a una persona se debe partir de que el mismo sea formado y valorado como persona y este en capacidad de asumir el reto de la totalidad.

El cuidado espiritual según Remen, sugiere que la curación no es una cuestión de mecanismos como los tratamientos y las medicaciones, sino más bien un trabajo del espíritu. Parece que en factor de curación existe un espíritu intrínseco del individuo. La salud espiritual se alcanza cuando una persona encuentra el equilibrio entre sus propios valores vitales, sus objetivos y sus creencias y los de los demás (13). Establecer una relación de cuidado con un cliente implica una interconexión entre la enfermera y el paciente. Esta es la razón por la que Watson describe la relación de cuidado en un encuentro espiritual. La espiritualidad ofrece un sentimiento de colectividad tanto intrapersonal como personal y transpersonal, conectado con lo invisible, Dios o poder superior.

En otra expresión de Talo se observa que verbaliza su percepción del arte de cuidar así: escuchar y hablar con el paciente, acompañarlo en el proceso de recuperar su salud, enseñarle para que pueda hacerlo por sí mismo. En lo expresado por la entrevistada se interpreta que ella anuncia tres características muy importantes en la actitud de cuidar: escuchar y hablar con el paciente, denota que ella entiende que cuidado empieza por la relación enfermera/paciente, lo cual es fundamental porque en ella se expresan sentimientos, emociones y la sensibilidad por el arte de cuidar. Segundo, se percibe que la actitud de la enfermera en el cuidado debe ser también de acompañamiento para no dejarlo solo en el dolor y tercero enseñarlo para el auto cuidado, a manera de evitar la dependencia, vale decir, acompañarlo en su recuperación con una visión de su futura reinserción a la familia. Una meta común del cuidado de enfermería relacionada con el mantenimiento o la restauración de la salud es ayudar a los pacientes a lograr un estado en donde ellos sean independientes. De allí, que gran parte de la práctica actual refleja una actitud de valor unida a la meta de independencia, e indica acciones de enfermería para ayudar a los pacientes a asumir su responsabilidad tan pronto como sea posible. Tanto el individuo sano o el enfermo anhela el estado de independencia. Tiene una serie de necesidades básicas para la supervivencia. Necesita fuerza, deseos, conocimientos para realizar las actividades necesarias para una vida sana e independiente. Las actividades que las 
enfermeras realizan para suplir o ayudar al paciente a cubrir estas necesidades es lo que Henderson denomina cuidados básicos de enfermería (4). Estos cuidados básicos se aplican a través de un plan de cuidados de enfermería, elaborado en razón de las necesidades detectadas en el paciente.

Se concluye que la característica fundamental de los modelos de cuidado que pertenecen a la escuela de las necesidades es que definen la acción de la enfermera como ayuda en la satisfacción de las necesidades fundamentales de la persona o del uso de sus capacidades para el auto cuidado.

\section{CONCLUSIONES}

El estudio del arte de cuidar permitió comprender el significado que le asignan al arte de cuidar en su desempeño cotidiano un grupo de profesionales de enfermería y de pacientes, además de comprender el sentido global de la práctica del cuidado y su complejidad. Todo lo cual nos permite asumir y sentirnos protagonistas como agentes de cambio de los modos de cuidar de nuestra profesión. Además, la investigación del cuidado de enfermería se enriquece con la producción del conocimiento, que la enfermera puede asumir y emplear para fortalecer el papel de cuidadora como acompañante en los procesos de desarrollo y crecimiento de la civilidad humana.

\section{REFERENCIAS BIBLIOGRÁFICAS}

1. BOFF. LEONARDO. Saber Cuidar. Etica do Humano-Compaixáo pela Terra. Brasil. Editora Vozes. 1999.

2. NIGHTINGALE. FLORENCE. Notas sobre Enfermería. Masson S.A.españa. Barcelona. 1990.

3. KOZIER y otros. Fundamentos de Enfermería. Conceptos, procesos y prácticas. Vol. 1. McGraw- Hill. Ed. Interamericana. Madrid. España. 1998.

4. ARENDT. HANNAH. La Condición Humana Barcelona. España. Paidos.1996

5. CARPER, B. A. Fundamental Patterns of knowing in nursing. Adv Nurs sci. 1978.

6. TORRALBA. I. ROSELLÓ, F. Antropología del cuidar. Ed.Vozes. Brasil, 2009.

7. SOURIAU, ÉTIENNE. Diccionario Akal de Estética. Akal, Madrid. 1998.

8. RITZER. GEORGE. Teoría Sociológica Contemporánea México, MCGRAW HILL. 1993.
9. TRAVELBEE J. Intervención de enfermería psiquiátrica: el proceso de la relación de persona a persona. Washington. O. P.S. 1 979.

10. BALDERAS PEDRERO MARÍA de la LUZ. Administración de los Servicios de Enfermería. México Mc. GRAW HILL. 2006

11. MAYEROFF, MILTON. A Arte de Servir ao Próximo para Servir a Sí Mesmo. Traductora: Cristina Carvalho Boselli. Editorial Record. Brasil. 1971.

12. MASLOW. A. H. A Theory of Human Motivation. Originall y Published in Psychological Review, 50. 1943.

13. POTTER. PERRY. Fundamentos de Enfermería. Editorial Harcourt, Madrid. España. 2001. 